\title{
Réflexions sur la réécriture en écriture d'invention
}

\section{François Le Goff}

\section{OpenEdition}

Journals

Édition électronique

URL : http://journals.openedition.org/recherchestravaux/325

DOI : 10.4000/recherchestravaux.325

ISSN : 1969-6434

\section{Éditeur}

UGA Éditions/Université Grenoble Alpes

\section{Édition imprimée}

Date de publication : 15 décembre 2008

Pagination : 19-34

ISBN : 978-2-84310-131-1

ISSN : 0151-1874

\section{Référence électronique}

François Le Goff, «Réflexions sur la réécriture en écriture d'invention », Recherches \& Travaux [En ligne], 73 | 2008, mis en ligne le 15 juin 2010, consulté le 10 décembre 2020. URL : http://

journals.openedition.org/recherchestravaux/325; DOI : https://doi.org/10.4000/recherchestravaux 325

(c) Recherches \& Travaux 


\section{Réflexions sur la réécriture en écriture d'invention}

Diversifier les pratiques d'écriture, aborder le littéraire par le faire, renouer avec une tradition rhétorique qui profiterait des acquis les plus récents concernant l'apprentissage de l'écriture sont autant de motifs de légitimation de l'écriture d'invention. Toutefois, l'intérêt que l'on peut porter à cet objet d'enseignement n'exclut pas l'examen critique, bien au contraire ${ }^{1}$. Et c'est par rapport à certains éléments de définition de l'écriture d'invention présents dans les textes officiels et à leurs applications dans les manuels et les épreuves anticipées du baccalauréat que je situerai tout d'abord mon propos.

En premier lieu, il me semble important de mettre en évidence la part prépondérante de l'argumentation dans les finalités de l'exercice, le formalisme dans la reproduction des discours de genre, et, par voie de conséquence, la fréquente artificialité des consignes d'écriture qui conduisent à privilégier des techniques narratologiques ou des procédés rhétoriques, déconnectés d'une réflexion sur le sens des écrits produits et sur les enjeux des apprentissages pour l'élève.

I. À propos de l'écriture d'invention, on pourra lire les analyses suivantes: C. BiseniusPenin, «Écriture d'invention au lycée et écriture à contraintes», Pratiques, n ${ }^{\circ}$ I 27-I 28, CRESEF, Metz, 2005, p. 209-229; B. Daunay, «Les ambiguiités des textes officiels sur l'écriture d'invention", Pratiques, n I 27-I 28, CRESEF, Metz, 2005, p. 17-30; A. Petitjean, «Histoire de l'écriture d'invention au lycée», Pratiques, n I I 7-I I 8, CRESEF, Metz, 2003, p. I 8 I-207; Y. Reuter, «L'écriture d'invention : réflexions didactiques sur une réforme en cours», Pratiques, n I 27-I 28, CRESEF, Metz, 2005, p. 7-16. 
En second lieu, la présentation qui est habituellement faite de cet écrit ignore le contexte d'apprentissage dans lequel il s'inscrit. Or, comme tout autre objet d'enseignement, l'écriture d'invention fait système, devient intelligible dans un contexte; son mode d'intégration à un projet d'enseignement englobant garantit ou non une exploitation optimale de ses potentialités de formation. Il est bien sûr rappelé dans le discours officiel que l'écriture d'invention n'est pas un objet isolé, aussi insiste-t-on toujours sur la nécessité du lien entre un texte-source et un écrit d'invention. Mais j'observe qu'une telle affirmation ne remet pas en question l'organisation générale d'une séquence d'apprentissage, pas plus qu'elle n'est une incitation à reconsidérer les rôles de chacune des sous-matières et activités du français dans l'appropriation des savoirs.

En effet, le fait de négliger la question des interactions lecture/écriture, de minorer une approche de l'écriture comme véritable lieu d'expérimentation et de construction de savoirs, tend à enfermer l'écriture d'invention dans deux conceptions combinées de l'écriture scolaire : une écriture modélisante et une écriture d'évaluation. Ramenée à sa définition officielle, l'écriture d'invention s'apparente alors à une chambre d'enregistrement de parcours de lecture au cours desquels des notions génériques, rhétoriques et stylistiques ont été repérées et analysées.

Or l'introduction de cette activité constitue, à mon sens, une opportunité pour revisiter l'articulation de l'écriture et de la lecture des textes relevant du champ de la littérature, pour développer des stratégies alternatives aux pratiques modélisantes et applicationistes ${ }^{2}$. L'innovation en didactique de l'écriture au lycée peut ainsi investir d'autres champs d'investigation inspirés des observations suivantes :

- la production d'écrits d'invention suppose un apprentissage continué de l'écriture;

- elle s'articule à l'ensemble des pratiques langagières, orales et écrites, à l'œuvre dans la classe et ne s'inscrit pas dans un seul type de relation entre lecture et écriture;

- elle occupe un temps spécifique et variable dans les activités de classe en

2. Est «applicationniste» l'attitude qui consiste à importer dans les disciplines scolaires les savoirs savants ou académiques, le plus souvent récents, sans aménagement particulier ni réflexion spécifique (par exemple, la théorie des ensembles utilisée pour produire les «mathématiques modernes» qui ont été abandonnés ensuite). C’est contre cette attitude qu'Y. Chevallard a proposé la notion de «transposition didactique» (Grenoble, La Transposition didactique, La Pensée sauvage, I985). Celle-ci implique une intégration raisonnée reposant sur une série d'opérations (choix, adaptation à des niveaux spécifiques, liaison avec les savoirs disciplinaires déjà là, avec des formes disciplinaires déjà installées comme les activités et exercices, les pratiques d'évaluation...). 
fonction des objectifs qui lui sont assignés et n'assume pas la seule fonction d'évaluation, en clôture d'une séquence.

À partir de ces postulats, je me propose d'examiner tout d'abord l'apport de la génétique textuelle à l'établissement d'une telle conception de l'écriture. Je poserai ensuite la question de la réécriture dans l'enseignement du français et ses possibles mises en œuvre. Un écrit d'élève de seconde, ayant pour objet la réalisation d'un incipit romanesque, brièvement commentée au cours de cet article, me servira d'illustration.

\section{La génétique textuelle ou la mise au jour du mouvement de l'écriture}

La génétique textuelle s'intéresse à ce qui demeure ignoré du lecteur d'une œuvre publiée, à ce qui forme le vaste ensemble des matériaux de la fabrique du texte. L'intérêt porté «aux résidus de la création ${ }^{3}$ » ne s'explique pas par la prétention illusoire à forcer les énigmes de la création artistique. Plus raisonnablement, Almuth Grésillon délimite le champ de la critique génétique en résumant ainsi ses trois fondements :

Son objet : les manuscrits littéraires, en tant qu'ils portent la trace d'une dynamique, celle du texte en devenir. Sa méthode : la mise à nu du corps et du cours de l'écriture, assortie de la construction d'une série d'hypothèses sur les opérations scripturales. Sa visée : la littérature comme un faire, comme activité, comme mouvement ${ }^{4}$.

La notion du faire, introduisant une réévaluation de la clôture du texte telle qu'elle a pu être posée par les théories textualistes, devient déterminante dans la compréhension des procédures de la production littéraire. La reconnaissance d'un artisanat de l'écriture conduit le généticien à ouvrir l'exploration de la genèse d'une œuvre à l'ensemble des matériaux et documents périphériques qui informent, directement ou non, du travail de l'écrivain. C'est ainsi que la constitution d'un dossier de genèse s'étend souvent au-delà de la seule réunion et mise en ordre des brouillons; les documents préparatoires, les carnets d'auteurs, la correspondance forment également une documentation précieuse dans le but d'enrichir la connaissance d'un projet littéraire.

En se fondant sur les archives rassemblées dans un dossier de genèse, la critique génétique ambitionne de montrer comment se nouent et se résolvent les tensions à l'œuvre dans un projet d'invention littéraire. Il va

3. D. Ferrer, «Le matériel et le virtuel : du paradigme indiciaire à la logique des mondes possibles", Pourquoi la critique génétique? Méthodes, théories, M. Contat et M. Ferrer éd., CNRS Éditions, I998, p. I3.

4. A. Grésillon, Éléments de critique génétique, lire les manuscrits modernes, PUF, I 994, p. 7. 
de soi que le manuscrit ne livre pas tous les secrets de l'origine d'une œuvre. Néanmoins, il peut donner à lire des signes de tous ordres, que le généticien classe, ordonne, relie afin d'éclairer ce qui demeure d'une énonciation in statu nascendi'. Corollairement à la notion du faire, s'impose l'idée d'une temporalité dans l'écriture, rarement compatible avec l'idée d'une progression linéaire et harmonieuse qui partirait d'un hypothétique germe fondateur, se déployant ensuite, intact, dans le cours de la rédaction, pour finalement trouver sa forme définitive et accomplie dans un état final indépassable. Le temps de l'énonciation, temps événementiel et profane de la genèse, est celui de l'accident, bien plus que celui de la fluidité. Une temporalité chaotique de la genèse, faite de fulgurances, d'arrêts, de détours, de reprises, institue une dynamique toujours particulière, ouverte à la concurrence des possibles, attestée par la présence des variantes d'écriture. La génétique accorde une égale attention à ces dernières, les traite sur le mode de la différence et de la variation et tient de ce fait à distance une lecture téléologique de la production littéraire. Au contact de ces variantes, on peut restituer, en assumant les failles et les trous d'ombre, la genèse du texte, ce dernier ayant pu subir d'incessantes transformations qui ont défiguré le projet initial.

Forte de ces hypothèses de recherche, la critique génétique déplace les centres de gravité de l'analyse littéraire : c'est le processus et moins le produit, l'énonciation et moins l'énoncé qui sont au cœur des préoccupations. Contrairement à la démarche philologique qui vise à établir, à partir des variantes, l'état estimé le plus vrai, le plus abouti ou le plus idéal du texte en fonction de la connaissance que l'on a de l'auteur et de ses écrits, la démarche génétique se focalise sur l'écriture en train de s'accomplir, sans faire du texte final celui par lequel tout état antérieur est évalué.

En proposant une sensibilisation au travail de l'écriture à travers la lecture des brouillons d'écrivains et des documents de genèse, les programmes de français au lycée ont marqué la volonté de faire évoluer la représentation que pouvait se faire un élève de seconde d'un texte littéraire et de montrer que celui-ci est soumis à un ensemble d'opérations qui font de lui un objet fabriqué. C'est ainsi que la connaissance des mécanismes de fabrication littéraire - exemples de la différenciation entre écriture à programmes et écriture à processus, de l'examen des différentes campagnes d'écriture et de leurs propriétés, des modes de sélection et de traitement de la matière fictionnelle ${ }^{6}-$ peut entrer dans un projet rédactionnel scolaire et accompagner l'élève dans

5. A. Grésillon et J.-L. Lebrave, «Les manuscrits comme lieu de conflits discursifs», dans C. Fuchs, A. Grésillon, J.-L. Lebrave éd., La genèse du texte : les modèles linguistiques, CNRS Éditions, I982, p. I 29-175.

6. P.-M. Biasi (de), La Génétique des textes, Nathan, 2000. 
la découverte d'une démarche d'écriture décomposée en différentes phases, au cours de laquelle la réécriture a toute sa place.

\section{La réécriture et l'enseignement-apprentissage de l'écriture}

\section{La polysémie du terme de «réécriture»}

Le terme de «réécriture» recouvre plusieurs types d'actions ayant en commun de marquer un retour sur un état d'écriture antérieurement produit ou de renouveler un même geste scriptural. La préfixation réunit ces actions autour des notions de récursivité et de duplication, sans pour autant parvenir à réduire la distance qui les sépare. Une écriture seconde peut présenter des écarts extrêmes par rapport à un état textuel antérieur sur lequel elle s'appuie : soit la réécriture est reproduction à l'identique d'un état antérieur, soit elle constitue une transformation complète de ce même état antérieur. Entre ces deux pôles, une infinie variation de degrés dans la transformation du texte existe, de la simple opération de modification locale d'un fait de langue au réaménagement de la globalité de l'énoncé... autant donc de processus récursifs qui n'ont ni la même puissance, ni la même signification et qui imposent nécessairement des clarifications en situation d'apprentissage scolaire.

\section{De la révision à la réécriture}

L'opération de révision est une activité cognitive que tout scripteur, expert ou novice, réalise dans le cours de la production, mais dont les manifestations graphiques varient considérablement. La lecture critique est censée permettre le repérage des dysfonctionnements et être suivie d'interventions assurant une amélioration de la production. On est alors dans une gestion idéale des procédures mais que dément souvent l'observation des pratiques effectives des apprenants7. Outre le coût cognitif élevé de la révision, ceci peut aussi s'expliquer par le fait qu'il est difficile pour un apprenti scripteur de devenir le lecteur critique de son propre texte et de se figurer mentalement l'état visé de son écrit.

Est-ce à dire que le principe d'un retour sur le texte est nécessairement condamné à l'échec et donc sans profit pour les apprentissages de l'écrit? Sans doute pas, d'autant que les stratégies de révision ne sont pas toutes équivalentes. Si la révision est motivée par le rétablissement de la norme, il va de soi que cela requiert de la part du scripteur un certain niveau de compétence,

\footnotetext{
7. M. Fayol éd., La production du langage écrit, PUF, I 996.
} 
une capacité à repérer dans son écrit les éléments défaillants. L'étendue de la révision est liée au degré de maîtrise langagière et à la représentation plus ou moins adéquate et conforme que le scripteur se fait de la production à réaliser. Cela signifie que l'élève peut réviser sa production sans en repérer les dysfonctionnements; il peut les percevoir sans toutefois disposer des compétences nécessaires pour les réduire; enfin, il lui arrivera aussi d'effectuer une modification qui changera la nature du dysfonctionnement sans toutefois l'effacer.

En revanche, dès l'instant où il est mis à disposition de l'élève des outils, c'est-à-dire des notions propres à la production de tel genre littéraire par exemple, on peut obtenir des révisions importantes. Prenons la consigne suivante :

Sous la forme d'un texte d'une trentaine de lignes, rédigez le début d'un récit dont un des personnages, un enfant, sera en position de victime. Le texte prendra la forme d'une scène romanesque.

L'écrit d'invention proposé aux élèves croise plusieurs objectifs que des lectures d'incipit romanesques ont permis de mettre en évidence. Il s'agit, à travers l'écriture, de poursuivre la réflexion sur la manière dont l'ouverture d'un récit établit un contrat de lecture et sur les fonctions du personnage dans la fiction. La notion de scène, rencontrée dans plusieurs des textes ${ }^{8}$, et dont les caractères ont été analysés, n'est pas maîtrisée dans le premier état:

\section{État I}

«Cela fait près de douze ans que Léo est né.

Il est né un $\mathrm{I}^{\text {er }}$ avril d'un père inconnu et d'une mère dépressive. Depuis sa naissance, il n'a vécu que chez ses grands-parents, qui avaient la fâcheuse tendance à être plutôt stricts, du genre pas trop indulgents. À chaque fois, il ne lui tardait plus qu'une chose, que sa mère vienne le voir. Seulement, le fait de la voir, le mettait dans un état de joie et d'enthousiasme et il ne se rendait pas compte du bazar monstre qu'il faisait, ce qui mettait sa mère en colère. Ce n'était même plus de la fureur mais de la rage, elle le balançait contre les murs... Résultat, des blessures à répétitions, fractures, hématomes....

Vers ses neuf ans, plainte de l'institutrice à la gendarmerie, la garde de Léo est enlevée à sa mère et à ses grands-parents.

Voilà en bref le résumé de sa petite enfance. Même s'il a souffert et si je ne sais comment il fait pour n'exprimer aucune rancœur envers les autres, il est jeune mais a déjà des convictions: la violence ne sert à rien et que l'indulgence ne coûte rien.

Le petit Léo est maintenant dans un foyer mais qui sait ce qu'il va devenir...»

8. Les seuils romanesques sélectionnés sont extraits des romans suivants : Madame Bovary de G. Flaubert, Le Naïf de Fr. Hellens, L'Enfant de J. Vallès, Le Sagouin de Fr. Mauriac, Génie la folle d'I. Cagnati. 
On s'éloigne de la notion de scène pour lire ce qui s'apparente à un sommaire. Cette configuration a alors un impact direct sur le processus de fictionalisation qui, ici, n'opère pas. Le récit relève plus du fait divers journalistique que du roman et se trouve empêché de construire un leurre crédible du personnage romanesque : le "petit Léo» n'accède pas à une existence fictionnelle. D'autre part, la durée de l'histoire racontée (de la naissance aux douze ans de l'enfant) et l'absence d'une localisation délimitée de l'action font que le lieu et le temps ne constituent plus des facteurs d'unité et de concentration dramatique.

Un projet de révision en différé, c'est-à-dire un retour sur l'écrit à un autre moment des apprentissages, portant sur la globalité du texte est nécessaire. Les modifications opérées au niveau de la forme vont avoir un impact sur le contenu même de la fiction. La réécriture (états 2 et 3) s'apparente à un nouveau démarrage :

\section{État 2}

Dans une petite exploitation de Bourgogne, loin de tout, parmi les vaches et les tracteurs, vivait un petit garçon qui se nommait Léo. Il était petit et pas bien gros mais respectueux. Il vivait seul dans cette ferme avec son grand-père, qui se chargeait de son éducation. Comme tous les jours, Léo devait se lever à l'aurore pour effectuer les travaux dans la ferme avant de partir à l'école et le soir en revenant, il devait encore traire les vaches. Quand il était épuisé, il se faisait insulter et même parfois taper. Quand Léo était à l'école, il s'endormait. Pourtant son plus grand rêve était de devenir professeur des écoles, mais pour son grand-père, c'était hors de question. Léo devait «reprendre» la ferme!

Un jour, l'instituteur alla voir le grand-père et lui expliqua que son petit-fils devait arrêter de travailler et se consacrer aux études car il pouvait aller très loin et qu'il fallait respecter le désir de Léo.

Son grand-père «monta sur ses grands chevaux» et expliqua à l'instituteur qu'il ne valait mieux pas qu'il monte la tête à son petit-fils....

Le soir venu, Léo rentra et alla traire les vaches. Victime de l'autorité de son grand-père, il prit une fessée et partit se coucher sans manger.

\section{État 3}

On frappa à la porte. Il entendit avec une certaine résonance le grincement de la porte et les quelques mots de politesse que connaissait son grand-père.

Intrigué, il sortit la tête de la cheminée qu'il était en train de ramoner. Il avait les mains et les habits tellement incrustés de suie qu'il n'imaginait même pas sa tête. Il s'accroupit en silence au bord de la cheminée et il regardait.

Un homme apparut; c'était son instituteur. Stupéfait, il regardait l'homme qu'il admirait le plus. Grand par sa taille mais aussi grand par sa générosité et son enthousiasme, vêtu de noir, coiffé d'un chapeau qui laissait échapper quelques cheveux blancs, il parlait à son grand-père. Il fit des efforts pour essayer d'entendre la conversation, lorsque son grand-père se retourna et l'appela. Il était assis dans le coin de la cheminée et se fit réprimander.

«Qu'est-ce que tu fais là, fainéant, espèce de carne, va traire les vaches, rentre les brebis, et occupe-toi des poules. Bon dieu, tu es vraiment un moins que rien!» 
Il passa à côté de son instituteur les yeux baissés, un peu honteux de ne pas lui avoir expliqué pourquoi il n'allait plus à l'école. Il regardait tellement le sol qu'il ne vit pas arriver le coup de pied sur ses fesses.

Dis bonjour! Impoli! Petit sauvage! Il dit donc bonjour avec un air désespéré, lui sourit et lut dans le regard de l'instituteur des encouragements et de la compréhension.

L'instauration d'un nouveau chantier d'écriture livre des savoirs et des compétences scripturales que le premier état n'avait pas réussi à mobiliser. L'exercice de la révision/réécriture ne se réduit pas seulement à un réglage du texte au niveau de la norme langagière, le plan de la signification importe tout autant. On peut ainsi avancer que l'opération combinant lecture et révision accroît son efficacité si tout ou partie des conditions suivantes sont réunies :

- la possibilité d'un investissement du sujet-scripteur dans ce temps critique de la révision, le plus souvent subi dans un enseignement traditionnel de l'écriture;

- l'engagement dans la révision visant une gestion globale du texte et provoquant de ce fait une reconfiguration significative du texte;

- la subordination de la révision à un enjeu de savoir ou de savoir-faire nouveau, ou non acquis qui, dès lors, institue la révision comme le seuil d'un nouveau projet rédactionnel.

\section{Un processus d'amélioration ou de variation de la production écrite?}

La réécriture se préoccupe de l'objet-texte, de son élaboration comme de son achèvement. Elle est associée le plus souvent à la recherche d'une réalisation jugée satisfaisante relativement à un ensemble de paramètres. Le bon sens ne peut que souscrire à cette mission de la réécriture : toute entreprise de remise sur le métier d'une production première est sous-tendue par l'espoir d'une réalisation meilleure. Or, cette perspective de la réécriture peut être discutée, pour au moins deux raisons.

La première me semble être contenue dans un échange entre Jean-François Halté, Claudette Oriol-Boyer et Jean Ricardou, au cours du colloque de Cerisy de r 979, «Pour un nouvel enseignement du français ${ }^{9}$ ». Au cours de l'échange, Jean-François Halté s'interroge sur les dérives contenues dans le terme d'amélioration avec cette idée d'«hypostasier un certain état final parfait du texte et [...] réintroduire la vieille problématique de la littérarité». Il poursuit en disant:

9. La revue Pratiques a consacré un numéro spécial à ce colloque organisé par J.-Fr. Halté et A. Petitjean : «Pour un nouvel enseignement du français (Colloque de Cerisy)», Pratiques, numéro spécial, CRESEF, Metz, I980. 


\section{RÉFLEXIONS SUR LA RÉÉCRITURE EN ÉCRITURE D’INVENTION}

Je vois se profiler une seconde menace derrière cette notion d'amélioration : celle d'un retour à l'idéologie formaliste de la clôture du texte car la bonne lecture devient celle qui serait capable de retrouver la totalité des règles - ce en quoi elle adopte la même démarche interprétative traditionnelle (qui cherchait l'épuisement du sens) que l'on croyait avoir évacuée ${ }^{10}$.

À ceci, Jean Ricardou répond que, si la notion d'amélioration doit être appliquée, ce n'est pas au regard de la valeur intrinsèque du texte, mais en fonction du degré de réponse positive qu'elle aura su apporter à la consigne de réécriture. L'amélioration n'est pas posée en tant que telle, mais dans une correspondance entre proposition critériée de réécriture et production nouvelle. Pour accepter l'idée d'amélioration, il convient d'admettre que tout retour sur un texte ne s'effectue qu'à travers l'explicitation d'un critère, à moins que l'élève ne fixe seul les motifs de la réécriture.

La seconde raison me semble plus décisive, car elle influence notablement une didactique de la réécriture en situation d'écriture d'invention. Le terme d'amélioration est concurrencé par celui de variation qui rend mieux compte du mouvement non linéaire de la genèse du texte. Quelque chose peut advenir dans un second état qui ne sera pas nécessairement meilleur (il faudrait ici définir les critères d'une qualité supérieure, ce qui ne serait pas sans difficulté), mais qui sera différent et à ce titre peut-être plus fécond, parce qu'il ouvrira de nouvelles voies que le projet initial n'avait pas imaginées. C'est pourquoi, quand la revue Le Français aujourd'bui se demande si l'amélioration est nécessairement l'horizon de l'acte de réécrire, Almuth Grésillon préfère, quant à elle, retenir une autre optique :

$[\ldots]$ «faire autrement», plutôt que «faire mieux». À l'école, on dit encore souvent à l'élève : «ce n'est pas bien, il faut faire mieux»; mais ce qu'on ne lui a pas permis de découvrir, c'est que réécrire peut être un jeu, que ce n'est pas tout de suite soumis à la notation, que c'est un plaisir. C'est pourquoi je préfère dire simplement qu'on revient sur ce qu'on a fait - dans le plaisir de créer de la variation, du multiple ${ }^{11}$.

Pour bien comprendre les implications de ces positionnements, il importe de différencier deux logiques qui déterminent les apprentissages, même si je crois qu'il est préférable de voir en elles des phénomènes de complémentarité et de translation plutôt que des territoires antagonistes.

ı. J.-Fr. Halté, «Analyse de l'exercice dit "la rédaction" et propositions pour une autre pédagogie de l'écriture», Pour une didactique de l'écriture, M. Charolles, J.-Fr. Halté et C. Masseron éd., p. 9-47, CRESEF, Metz, i 989, p. 44.

I I. J. Authier-Revuz, A. Grésillon, «La réécriture. Questions théoriques. Interview croisée de J. Authier-Revuz et A. Grésillon», Le Français aujourd'bui, n I44, AFEF, 2004, p. I 2. 
En effet, une première option de la réécriture consisterait à valoriser le traitement de l'erreur relevée dans un état initial. À quelque niveau que ce soit de la production, l'élève peut être amené à reconsidérer son énoncé parce que des dysfonctionnements ont été signalés. Formes morphosyntaxiques défaillantes, segments textuels agrammaticaux, système énonciatif défectueux, les zones textuelles de reprise sont aussi nombreuses que les membres qui construisent le discours. De façon générale, l'amélioration dans cette configuration peut être validée, soit parce que la réécriture a permis de rétablir la production dans une forme objectivement plus acceptable en référence à une norme langagière, soit parce qu'elle retrouve le chemin du projet initial.

Dans les états 2 et 3 de l'écrit d'invention cité plus haut, un système du récit en accord avec une inscription générique de convention se met en place. L'effacement des embrayeurs atteste l'adoption d'une entrée dans le régime de l'histoire par opposition à celui du discours. Cette réorientation énonciative est décisive car elle ouvre la voie au redéploiement de la fiction, sans que la voix de l'énonciateur ne s'interpose entre elle et son lecteur. Dans un lieu à présent situé géographiquement («une petite exploitation de Bourgogne»), le personnage s'étoffe grâce à une série d'actions dont il est l'agent. Par rapport à l'état I, sa position dans la fiction évolue donc sensiblement; surtout, il n'est plus un concentré de la souffrance mais une figure romanesque qui est aussi animée par un désir (être professeur des écoles), ce qui justement introduit un conflit avec son entourage (le grand-père). La signification que l'on peut prêter au récit évolue aussi. En faisant en sorte que le personnage ne soit plus enfermé dans une représentation stéréotypée et surexposée de la victime, le récit installe de nouveaux possibles narratifs, reconnait à l'histoire un futur et assure de ce fait les conditions favorables à l'établissement du suspense qui faisait défaut précédemment. À l'exposition sans partage du malheur, se substitue un programme narratif articulé autour de la problématique de la succession et de l'héritage social et culturel; l'enfant s'oppose à l'aïeul et trouve dans l'instituteur un allié. La dynamique du conflit entre dans la composition d'une mise en intrigue, absente du premier état.

La seconde option est moins préoccupée de l'erreur que de la propension de la réécriture à permettre l'émergence de solutions nouvelles dans la manière de mettre en mots l'idée. Il peut s'agir d'une simple opération de substitution construite sur le principe de l'équivalence sémantique; mais cela peut aussi être accompagné d'un mouvement amplifié de textualisation qui introduit de nouvelles strates dans le discours. De façon plus ou moins radicale, un autre projet s'impose : les études génétiques montrent justement comment les modifications successives à l'œuvre dans les brouillons 
d'écrivain ont cette capacité à redéployer un projet liminaire ${ }^{12}$. La réécriture, particulièrement quand on se place dans une démarche différée et multiple de retour sur une production, va porter plus loin cette potentialité du geste scriptural. La réécriture envisagée de cette manière déborde le cadre d'un projet de réduction de l'erreur ou d'une narratologie appliquée et s'apparente plus à une aventure du scripteur, à la fois dans la langue et dans son expérience de sujet ${ }^{13}$.

\section{Réécriture et écriture d'invention}

À ma connaissance, la réflexion sur la réécriture, ainsi que les protocoles expérimentés dans les classes et exposés dans les différents travaux de recherche, ont concerné de façon quasi exclusive le premier degré et le collège $\mathrm{e}^{14}$. Centrée surtout sur les apprentissages fondamentaux, la maitrise du récit et les types textuels qui le composent, la réécriture, en tant que dispositif d'enseignement/apprentissage, semble bien disparaître au lycée. Dans la culture de l'institution scolaire et en dépit des démentis apportés par la réalité vécue dans les classes, survit l'opinion selon laquelle l'élève entré en seconde est censé posséder une maîtrise suffisante de l'écrit, les objectifs du lycée se situant surtout au niveau de la fréquentation des textes littéraires et des savoirs y afférents. Par ailleurs, la représentation dominante des écrits de savoirs du type commentaire littéraire ou dissertation n'a pas véritablement encouragé le développement d'un apprentissage de l'écriture au lycée : l'écrit remplit en premier lieu une fonction de médiation sous une forme normée, au service de notions construites antérieurement, dans le temps des lectures. Pourtant, la réécriture du texte d'élève intégrée à un projet de séquence de lecture du texte littéraire mérite à plus d'un titre d'être considérée.

\section{Modifier le rapport à l'écriture de l'élève}

Du point de vue des représentations et des postures de l'apprenant visà-vis de la pratique scripturale, l'expérience montre que la réécriture permet de construire une posture d'auteur, conscient des effets pragmatiques que les

I 2. M. Collot, La Matière-émotion, PUF, I997; P.-M. Biasi (de), «Flaubert, la genèse d'un incipit. Écrire la première phrase de La Légende de saint Julien l'Hospitalier», Équinoxe, $\mathrm{n}^{\circ} \mathrm{I} 6$, Kyoto, Rinsen-Book Co, I 999, p. 68-8I.

13. Cette option est présentée dans Fr. Le Goff, «Réécriture et écriture d'invention: l'exemple de la fable», Pratiques, n I 27-I 28, CRESEF, Metz, 2005, p. I 83-208: le genre de la fable est découvert à travers une succession de variantes.

I4. D. Bessonnat, «Deux ou trois choses que je sais de la réécriture», Pratiques, n 105-106, CRESEF, Metz, 2000, p. 5-22; D. Bucheton, Écritures, réécritures : récits d'adolescents, Bern, Peter Lang, 1995 ; C. Masseron, «Entre argumentation et langue : propositions visant à la réécriture de fragments très locaux», Pratiques, n 84, CRESEF, Metz, I 994, p. 4I-54; S. Plane, «Écriture, réécriture et traitement de texte», dans J. David et S. Plane éd., L'Apprentissage de l'écriture de l'école au collège, PUF, i 996, p. 37-77. 
différents états d'écriture provoquent, et de faire évoluer ses représentations concernant les processus de l'écriture ${ }^{15}$. Introduire une régularité de la réécriture est un moyen pertinent pour interroger certains dogmes, largement partagés par les élèves de lycée :

- un dogme conceptuel qui réduit l'écriture à un objet de médiation, au service de l'expression et de la représentation;

- un dogme cognitivo-scriptural qui entérine l'idée que la pensée précède l'écriture et que l'écriture n'est qu'une opération de mise en mots d'un déjàlà, préconçu intellectuellement;

- un dogme esthétique qui reconnaît les qualités formelles d'une production écrite en fonction de critères normatifs qui fondent le bien-écrire;

- un dogme artistique qui interprète la singularité, l'originalité d'un écrit comme étant l'expression du don et de l'inspiration, et non le résultat d'un travail et des influences intertextuelles;

- un dogme téléologique qui valorise le produit achevé et moins le processus de génération de l'écriture.

\section{Construire des compétences sur l'écriture du genre littéraire}

Au lycée, les programmes actuels renforcent la voie d'accès à la littérature par l'étude des genres. Si celle-ci n'a jamais été absente des enseignements littéraires, elle apparait aujourd'hui plus systématique, ne serait-ce que dans la manière dont est organisée la lecture des œuvres. Ces dernières sont réparties en objets d'étude qui se réfèrent explicitement à des catégories génériques : le récit, la comédie ou la tragédie, l'éloge et le blâme en seconde; le roman, l'autobiographie, les formes littéraires de l'argumentation, la poésie, l'épistolaire en première.

L'approche des genres doit ainsi permettre, selon le discours officiel, une connaissance de la littérature, des liens comme des différences entre texte littéraire et non littéraire, une connaissance de leurs codes et de leurs règles de fonctionnement et au-delà une appréhension des esthétiques qui les constituent et de leurs modes de subversion ou de recréation. Dans le champ de la lecture littéraire, le genre voit sa fonction d'outil heuristique confortée. Une œuvre est porteuse d'un ensemble hétérogène de signes manifestes ou latents, externes ou internes qui prédisposent le lecteur à un certain type de réception. Un énoncé est ainsi diversement reçu selon qu'il est identifié comme relevant du roman sentimental, du conte ou encore de la poésie lyrique.

Mais l'importance accordée aux genres dans l'enseignement de la littérature a aussi ses revers. L'hyper-visibilité de la notion de genre et la manière

I s. En plus de l'article cité à la note i 2, voir aussi Fr. Le Goff, «Variantes et variations discursives en écriture d'invention», dans J.-M. Pottier éd., "Seules les traces font rêver» Enseignement de la littérature et génétique textuelle, SCEREN - CRDP Champagne-Ardenne, 2006, p. 213-222. 
dont elle structure les épreuves du baccalauréat et réglemente la lecture des textes littéraires créent des déséquilibres dans la façon de concevoir le rôle de la littérature dans la formation du lycéen. La démarche d'analyse dominante à laquelle participe le genre renvoie le plus souvent l'expérience du lecteur dans les marges. La prégnance des savoirs spécialisés profite à une description objective et quelque peu désincarnée des fonctionnements textuels ${ }^{16}$. Sous les pressions multiples du curriculum officiel, des manuels et des épreuves anticipées du baccalauréat, les textes sont d'abord considérés comme des exemplaires de genre dont on exhausse les caractéristiques génériques pour tenter de construire une interprétation. En laissant libre cours à un nouveau formalisme, on perd alors de vue l'équilibre revendiqué par les programmes, à savoir le développement d'une conscience historique fondée sur une contextualisation des œuvres, une réflexion sur leur environnement esthético-culturel et l'importance de la relation singulière que le lecteur entretient avec une œuvre.

Sur le versant de l'enseignement/apprentissage de l'écriture et en liaison avec les activités de lecture, la notion de genre a, tout au long de ces dernières années, fortement inspiré les travaux didactiques. Sous l'influence de la linguistique appliquée et de la sémiotique textuelle, on élabore des matrices textuelles dans des phases d'observation/reproduction. Les constituants d'un genre deviennent ainsi des contraintes productives, dans une démarche générale de rationalisation des opérations scripturales. La thèse défendue par Karl Canvat reflète alors bien la conception dominante de l'acquisition du savoir-écrire quand il affirme :

[1] n'est pas de pratique scripturale qui ne se situe en fonction d'un système générique préexistant, que ce soit pour le respecter ou pour le transgresser. Plus précisément, la dimension relativement normative-prescriptive du genre fait de lui une unité de structuration, d'organisation et de composition qui facilite les guidages, globaux ou sectoriels, de l'écriture ${ }^{17}$.

On le voit, l'articulation lecture/écriture, la référence à des modèles de genre, la production outillée du texte forment, dès le collège, un socle notionnel et procédural dans l'appropriation écrite des genres du discours. Nous les retrouvons au lycée mais dans une approche complexifiée qui se traduit par une demande de restitution de savoirs littéraires en situation de production.

I6. G. Langlade, «La littérature restreinte de l'enseignement des lettres», Le pari de la littérature, quelles littératures de l'école au lycée?, A. Brillant-Annequin et J.-Fr Massol éd., CRDP de l'académie de Grenoble, 2005, p. 5I-62.

17. K. Canvat, «La notion de genre à l'articulation de la lecture et de l'écriture», dans Y. Reuter éd., Les Interactions lecture-écriture, Actes du colloque Théodile-Crel (Lille, nov. 1993), Bern, Peter Lang, I 998, $2^{\mathrm{e}}$ éd., p. 275. 
Mais, s'il est pertinent de ne pas séparer les énoncés des genres de discours dans lesquels ils sont produits, il est en revanche réducteur d'enfermer l'exercice de l'écriture d'invention dans un face-à-face du texte et du genre. L'effet le plus immédiat est de conforter une conception formelle de l'écriture, tout entière dévolue à la mise en œuvre des contraintes génériques. L'applicationnisme devient la règle, à l'image de ce qui se produit aussi, d'ailleurs, dans le cadre de la lecture analytique des textes littéraires.

Comment, dans ces conditions, se situe la réécriture du texte d'élève par rapport au genre? Le premier point réside dans la volonté de ne pas faire du genre l'horizon indépassable de toute pratique d'écriture. En fonction de l'appartenance générique du texte à produire, une sélection ajustée des compétences langagières doit être mise en place. Tel projet peut requérir une étude plus systématique, par le biais des réécritures successives, de la structure textuelle, tel autre peut conduire à une utilisation plus complexe des marques de l'énonciation ou du traitement de la temporalité dans le discours.

Cependant, il me semble aussi essentiel qu'une approche grammaticale soit coordonnée aux effets de sens générés par le texte. Aussi les considérations langagières ont-elles intérêt à fonctionner en rapport étroit avec une problématique littéraire ou un projet d'expression du sujet-scripteur. Par exemple, en privilégiant l'instauration de situations-problèmes, on travaille, dans un cadre fixé par le genre, à l'émergence et à la compréhension des modes de représentation de la référence dans le champ de la fiction ou du récit de vie, des régularités et stratégies énonciatives impliquées par le genre, enfin, des codes en exercice dans le champ de la communication littéraire.

C'est ainsi que l'état 3 de notre exemple ne se limite pas à une révision formelle; sa visée réparatrice occasionne d'autres effets pragmatiques. Avec l'effacement du sommaire, on assiste à un allègement de la pression informative et de la tonalité dramatique. L'insistance à provoquer l'empathie du lecteur vis-à-vis du personnage par la relation d'actes de soumission à l'autorité du grand-père est résorbée en une situation événementielle unique qui pose la relation de l'enfant à l'adulte mais sans ostentation. C'est à présent un faisceau de signes sémantiques, discursifs, actantiels qui fonde la cohérence de la scène. La signification du récit passe par d'autres relais textuels, élargit les lieux d'investissement possibles du lecteur, complexifie son parcours de lecture. Enfin, l'exploitation de la focalisation interne est une réponse à cette volonté, rappelée dans les séances collectives de lecture et au cours de l'accompagnement des élèves dans le travail de réécriture, de différer l'information, ou du moins de ne la livrer que partiellement pour permettre au lecteur de faire son propre chemin, ce qui avait été admis comme un motif supérieur d'implication dans 
la fiction... sans compter que le point de vue interne est souvent interprété comme un facteur d'accroissement de l'empathie du lecteur.

En favorisant l'idée d'une fabrication du texte dans un mouvement de réglages successifs et d'explorations de pans de la fiction qui adviennent dans le processus d'écriture, on tâche de convertir la commande magistrale en projet de l'élève. Car c'est à lui que revient la responsabilité de composer une unité textuelle qu'un lecteur saura reconnaitre et pourra investir en faisant jouer sa part collaborative. L'acte d'écrire progresse dans une perspective du lire; ce qui se construit dans l'écriture, c'est aussi un lecteur.

\section{Adopter d'autres formes d'aide à l'écriture et d'évaluation}

Du point de vue de l'activité enseignante, compte tenu du fait que l'écriture d'invention est envisagée comme la mise en œuvre de savoir-écrire décomposables, faisant appel à un ensemble de compétences de nature et de complexité variées, on assiste à un réaménagement des habitus professionnels. Le principe d'un étalement temporel de l'activité scripturale modifie la fréquence, la nature et le rôle des interventions du professeur. Signalons, par exemple, une diversification des modalités d'interaction entre lecture et écriture, et des types d'évaluation, la mise en œuvre de situations de remédiation et d'interaction entre pairs.

Introduire une pratique de la réécriture en situation d'écriture d'invention, cela signifie aussi rompre avec la prédominance de l'évaluation certificative. Tout en demeurant dans un cadre institutionnel qui attend du professeur une évaluation régulière des acquis et sa traduction chiffrée, il s'agit plutôt de recourir à une évaluation formative, au sens que lui prête notamment Philippe Perrenoud : est «formative toute évaluation qui aide l'élève à apprendre et à se développer, autrement dit, qui participe à la régulation des apprentissages et du développement dans le sens d'un projet éducatif ${ }^{18} \gg$. Le caractère ouvert de l'évaluation formative, sa souplesse d'exploitation se traduisent dans les faits par sa présence à différentes étapes de la séquence, sans l'obligation d'une élaboration ambitieuse, qui viendrait alourdir un projet de lecture/écriture. Les intervalles ménagés entre les productions sont l'occasion de porter à la connaissance des élèves des compétences non atteintes ou nécessitant des ajustements ou bien des approfondissements. Qu'elles soient d'ordre déclaratif (on pense aux savoirs littéraires) ou procédural (les actions mobilisées par le savoir-écrire), leur caractère évaluable permet la tenue de phases de régulation, de verbalisation et de reformulation des obstacles rencontrés.

I8. P. Perrenoud, «Pour une approche pragmatique de l'évaluation formative», Mesure et évaluation en éducation, $\mathrm{n}^{\circ}$ I3-14, ADMEE, Québec, r 99I, p. 50. 
Enfin, du point de vue de l'évaluation en tant qu'attribution d'une valeur à une production, il est manifeste que la réécriture modifie le regard porté sur les performances de l'élève. La confrontation des états permet de prendre en considération l'évolution d'un écrit d'invention. Elle sert à apprécier la façon dont l'élève s'est employé à résoudre les dysfonctionnements d'un état antérieur ou la façon dont il a intégré une nouvelle consigne d'écriture censée transformer sa production. Le jugement porté sur un état d'écriture est modalisé par une évaluation globale du projet et par l'appréciation - certes subjective mais que l'on ne saurait écarter - de l'investissement de l'élève dans son travail.

En permettant un retour sur l'écrit, il est donné à l'élève la possibilité de comprendre en situation réelle et active de production que la révision ne consiste pas simplement à rendre le texte conforme mais peut aussi provoquer une distribution inédite des contenus, voire l'introduction de nouveaux éléments de contenus.

Une démarche de reformulation globale d'un texte en situation de réécriture différée privilégie une conception de la réécriture qui assure plusieurs missions : elle intervient tout d'abord en tant qu'élément organisateur d'un projet d'écriture; elle fixe des objectifs de maitrise de la langue en termes de variation discursive; enfin elle s'impose comme un outil d'intégration de savoirs littéraires par l'exercice du faire. C'est à ces titres qu'elle peut être amenée à jouer un rôle dans un enseignement de la littérature au lycée associé à la pratique de l'écriture. 